\title{
Determinants of presence in 3D virtual worlds: A structural equation modelling analysis
}

\author{
Meyrick Chow \\ Tung Wah College
}

There is a growing body of evidence that feeling present in virtual environments contributes to effective learning. Presence is a psychological state of the user; hence, it is generally agreed that individual differences in user characteristics can lead to different experiences of presence. Despite the fact that user characteristics can play a significant role in research on presence, most studies on the determinants of presence focus on immersive, interactive, and perceptually realistic aspects within the technology. Research into user characteristics and how they affect presence has received little attention thus far. The purpose of this study is to explore how user variables combine and interact to predict the level of presence in a 3D virtual learning environment. One hundred and eighty-five nursing students participated in this study and completed a questionnaire measuring user variables. Structural equation modelling was employed as the main technique for data analysis to ensure methodological rigor. The findings indicate that subjective norm, perceived ease of use, computer self-efficacy, and perceived usefulness account for $52 \%$ of the total variance of the sense of presence. This study makes a significant contribution by developing and validating an initial model of the determinants of presence in a 3D virtual world.

\section{Introduction}

During the past decade, interest in the educational use of three-dimensional (3D) virtual worlds has been growing substantially (Bulu, 2012). 3D virtual worlds are customised settings that may be modelled according to those in the real world or may be fantasy based. They can be defined as "a synchronous, persistent network of people, represented as avatars, facilitated by networked computers” (Bell, 2008, p. 2). An avatar is a virtual body and is controlled by the user. Avatars may move freely in the virtual world by walking, running, flying, or teleporting from one location to another, and may manipulate objects. They may stand up or sit down in various poses, dance with someone, and make human-like gestures (Johnson, Vorderstrasse, \& Shaw, 2009). Virtual worlds offer a platform not only for education, but also for communication, business, and organisational development. Virtual worlds merge many qualities of the Web such as online games, social networking, usergenerated content, creativity applications, and telecommunication technologies (Schmidt \& Stewart, 2010). They provide users with the opportunity to meet individuals from around the world and to share ideas in a 3D environment.

Representation through avatars, communication tools for interaction, and three-dimensional immersive environments are the important features of 3D virtual worlds (Bulu, 2012; Girvan \& Savage, 2010). These features make people, places, and things in virtual worlds concrete and tangible, and give users the perception that they are really experiencing the environment. The experience of presence is a positive attribute of a technology and, hence, has great relevance to the design and evaluation of a broad range of media products for use in, for example, simulation training in education, and telemedicine in health care (Lee, 2004). Indeed, the field of presence research has grown substantially over the past few years. Factors that may affect the experience of presence have been suggested in the literature. However, how these factors may combine and interact to influence presence is not clear. The purpose of this study is to fill some of the gap in the research on determinants of presence, by examining the level of presence in a virtual world that students experience, and how factors combine and interact to predict the students' experience of presence. 


\section{Literature review}

\section{The concept of presence}

Since Minsky coined the term telepresence in 1980, scholars from various fields have suggested an abundance of conceptualisations of the sense of presence. Lombard and Ditton (1997) reviewed a broad body of literature related to presence and identified six different explications of presence: social richness (the extent to which the medium is perceived as personal, sociable, sensitive, or warm), realism (the extent to which a medium can seem perceptually and/or socially realistic), transportation (the sensation of "we are together," "you are there" and/or "it is here"), immersion (the extent to which the senses are engaged by the medium), social actor within medium (the extent to which users respond socially to representations of people through a medium), and medium as social actor (the extent to which the medium itself is perceived as a social actor, for example, treating computers as social entities). Based on the commonalities between these different conceptualisations, Lombard and Ditton defined presence as the extent to which a person fails to perceive or acknowledge the existence of a medium during a technologically mediated experience, or "the perceptual illusion of non-mediation" (p. 9).

The existence of six different conceptualisations of virtual experience calls for a multidimensional approach to the sense of presence. After an extensive explication process, Lee (2004) defined presence as "a psychological state in which virtual objects are experienced as actual objects in either sensory or nonsensory ways" (p. 27). He then defined three types of presence based on the general definition of presence and the corresponding domains of human experience in virtual environments - physical, social, and self presence. Physical presence refers to a psychological state in which virtual physical objects are experienced as actual physical objects in either sensory or nonsensory ways. Put differently, physical presence occurs when a technology user does not notice either the para-authentic nature of mediated objects (or environments) or the artificial nature of simulated objects (or environments). Social presence refers to a psychological state in which a virtual social actor is experienced as an actual social actor in either sensory or nons-ensory ways. In other words, social presence occurs when a technology user does not notice the para-authenticity of mediated humans and/or the artificiality of simulated non-human social actors. Lastly, self presence refers to a psychological state in which a virtual self is experienced as the actual self in either sensory or nonsensory ways. Self presence occurs when a technology user does not notice the virtuality of either para-authentic representation of his/her own self or artificially constructed self inside a virtual environment (Lee, 2004).

The proliferation of analyses and multidisciplinary discourses in the literature has not led to a standard, universal definition of presence. Yet there is an overall agreement among researchers of presence that the concept of presence has multiple dimensions, and that flexibility and fluidity are needed to situate a definition of presence in relation to the context (Lee, 2004; Tamborini \& Skalski, 2006). The choice of presence dimension should depend on the type of technology under investigation and other variables of interest. Given the increase in visual representations of self in avatar-based virtual worlds and the fact that physically and socially appropriate responses to the input of users in virtual environments can enhance the feeling of self presence, the focus of the current study is on self presence (Lee, 2004) in a 3D virtual world and on examining factors that influence the perception of self presence.

Presence is a human reaction to immersion (Slater, 2003). Immersion is an objective description of aspects of the system such as display resolution and field of view that facilitate the production of multimodal sensory input to the user (Slater \& Wilbur, 1997). In general, the more immersive a virtual environment is, the greater feeling of presence a technology user tends to experience in it (Schuemie, Van Der Straaten, Krijin, \& Van Der Mast, 2001). However, there is a debate that desktop virtual worlds are non-immersive and hence incapable of generating presence (Blascovich \& Bailenson, 2011; Winn, 1993). Although a fuller immersion in virtual reality should trigger a stronger feeling of presence, it does not mean that the only way we can achieve presence is through immersive virtual reality. Lombard and Ditton's (1997) definition of presence also includes traditional media such as the radio, television, and film that might offer a lesser degree of presence, but still trigger the illusion that the mediated experience is not mediated, but real (Romano \& Brna, 2001). It is possible to reach different forms of presence using different types of virtual environments in each situation. The visual and 
physical realism of desktop 3D virtual worlds that adds to the virtual space combine to produce a profoundly immersive experience can convey a feeling of being there and a strong sense of social presence when other avatars are present. The immersive nature of 3D virtual worlds, crossing physical, social, and cultural dimensions, can provide a compelling educational experience.

\section{Presence and learning in virtual 3D environments}

Virtual worlds are very attractive and are being used in numerous universities to provide learning experiences and conduct online classes. In a 3D virtual environment, students are free to explore. They allow the teacher to become the mentor or the guide, not the broadcaster (Welch, 2008). In virtual worlds, learning is done more by participating and doing than by listening and absorbing. Users can use their avatars to carry out activities that might be impossible in the real world. For example, students in healthcare disciplines are required to engage in experiential learning activities by participating in clinical placements. However, several factors prohibit them from obtaining much clinical learning and practice. These include the presence of a large number of students, which limits the actual amount of time that the students are exposed to patients; an emphasis on patient safety; medical errors; ethical considerations; pandemics; and crisis situations. Therefore, educators need to bring in simulation activities to reproduce real-life situations for students to learn and develop clinical skills and concepts. Virtual worlds can replicate real-world learning experiences previously available only through faceto-face encounters. They help students to bridge the gap between knowledge and application by providing them with opportunities to use simulation in a safe environment, and by allowing individuals to practise skills, try out new ideas, and learn from their mistakes without disadvantage (Skiba, 2009). Having gone through these experiences, students can have relatively safe clinical experiences in their placements (Schmidt \& Stewart, 2010). In addition, the simulation activities can enhance critical-thinking skills and allow students to take risks and make decisions independently. Students are able to access and repeat practices in virtual worlds as many times as they desire, enabling them to self-direct their learning until such time that they feel comfortable with the procedure and can practise without harm to patients (Schmidt \& Stewart, 2010). For example, Chow, Herold, Choo, and Chan (2012) designed and developed a virtual ward for practising rapid sequence intubation (RSI) on the campus of a university in the 3D online world Second Life (SL). RSI involves the rapid administration of a sedative agent and a neuromuscular blocking agent to a patient to induce unconsciousness and motor paralysis for tracheal intubation. To practise the skill of RSI, students entered a standard treatment room with a patient figure lying on a bed, a range of medical equipment ready to be used, as well as a doctor and a nurse already in the room. The simulation followed a number of possible, but strictly scripted paths based on the actions and responses of student avatars. Once the student had completed the entire procedure in the virtual ward, the system would automatically generate a record of the student's performance. Virtual worlds have been widely accepted and used in healthcare, for example, in disaster simulation, and in nursing, medical, and nutrition education (Chow et al., 2012; Kamel Boulos, Ramloll, Jones, \& Toth-Cohen, 2008; Schmidt \& Stewart, 2010).

There is a growing body of evidence that feeling present in virtual environments is one of the factors that contributes to effective learning. For example, in a 10-year review of empirical research on the educational applications of virtual reality environments, Mikropoulos and Natsis (2011) found that at least 12 out of the 53 studies that were reviewed explored the sense of presence, and positively connected presence with learning. Similarly, a systematic evaluation of evolving educational virtual environments revealed that levels of presence were generally correlated with the achievement of learning objectives (Selverian \& Hwang, 2003). In investigating the effect of presence on learning outcomes in virtual reality environments, Mikropoulos (2006) found that in the learning of history the existence of avatar-enhanced presence helped learners to complete their learning tasks more easily and successfully. The sense of presence that learners feel is of major importance in learning because it not only enhances first-hand experiences, but also enables first-person psychological activity to occur when learners interact directly with virtual worlds (deByl, 2009). In applied, situated virtual worlds, the sense of presence contributes more to learning than just cognitive gain - it also nurtures an intrinsic motivation to learn for its own sake, without extrinsic rewards. 


\section{Determinants of presence}

The determinants influencing the subjective feeling of presence can be classified into two general categories of variables: (1) media characteristics and (2) user characteristics (Galloso, Feijoo \& Santamaria, 2015; Ijsselsteijn \& Riva, 2003; Ribbens \& Vanden Abeele, 2008). Media characteristics can be subdivided into aspects of media form and media content. Media form is the physical, objective properties of a display medium. It is related to the extent and fidelity of sensory information presented, the match between a user's actions and the perceptible spatial-temporal effects of those actions, and the ability of a technology to enable the user to interact with the content and to modify it. Media content refers to the activities, objects, characters, messages, or story depicted via a technology. The elements of media content that may determine presence are the representation or virtual body of the user in the virtual environment, social realism, autonomous behaviours, and appearance of characters and objects. Presence is a psychological state of the user; hence, it is generally agreed that individual differences in user characteristics can lead to different experiences of presence (Ijsselsteijn, deRidder, Freeman, \& Avons, 2000; Tamborini \& Skalski, 2006). The feeling of presence has been found significantly influenced by the cognitive, emotional, and motivational factors of media users, such as immersive tendency, attention, skill, perceived control, personality traits, and spatial intelligence (Galloso et al., 2015; Weibel, Wissmath, \& Stricker, 2011).

Despite the fact that user characteristics are likely to play a significant role in research on presence, most studies on the determinants of presence focus on immersive, interactive, and perceptually realistic aspects within the technology. Very little research has been done on the user determinants of presence in virtual environments since Alsina-Jurnet and Gutierrez-Maldonado's (2010) article, which investigated five user characteristics (test anxiety, spatial intelligence, verbal intelligence, personality, and computer experience) on the feeling of presence. The essence of presence cannot be grasped by taking only the realism or fidelity of the mediated environment into account. A larger emphasis on user variables could benefit research dealing with presence, since acceptance and uptake of the technology will depend to a large extent on the experiences and responses of users towards the technology (Ijsselsteijn \& Riva, 2003). Clearly, there are many user characteristics that may have an impact on the extent to which a user experiences presence. The purpose of this study is to explore how variables combine $\backslash$ and interact to predict the level of presence in a 3D virtual learning environment.

\section{Methodology}

\section{Virtual world environment}

SL is a 3D virtual world created by Linden Lab, a US company. It is an online persistent space totally created and evolved by its users. Many companies and organisations have an online presence in SL. In SL, users can do a lot of things, such as promote charitable organisations and political philosophies, go to live concerts and social gatherings, or attend press conferences and college classes. Though not primarily developed for education, it is widely used to support teaching and learning activities (Chow et al., 2012; Dalgarno, Lee, Carlson, Gregory, \& Tynan, 2011; Jennings \& Collins, 2007). In SL, users may use avatars to explore the virtual environment and interact with one another through instant messaging, texting, and voice communication (Schmidt \& Stewart, 2010). SL provides users with a virtual environment within which they can participate in experiential learning and practise their skills in realistic simulations.

In this study, we designed and developed a virtual classroom in SL for nursing students to learn about personal protective equipment (PPE) (Figure 1) and virtual hospital wards (Figure 2) for them to practise the selection and use of PPE. PPE is specialised clothing or equipment worn by healthcare workers for protection against infectious materials (Siegel, Rhinehart, Jackson, Chiarello, \& the Healthcare Infection Control Practices Advisory Committee, 2007). PPE prevents a healthcare worker from coming into contact with an infectious agent or body fluid that may contain an infectious agent by creating a barrier between them. PPE may include items such as gloves, gowns, protective eyewear, masks, respirators, and caps. These items are used alone or in combination to protect mucous membranes, airways, skin, and clothing from coming into contact with 
infectious agents. Selecting appropriate PPE items in clinical practice requires an assessment of the level of risk associated with a specific patient care activity or intervention and the modes of disease transmission (Casanova, Alfano-Sobssey, Rutala, Weber, \& Sobsey et al., 2008; Department of Health, 2001; Siegel et al., 2007). Moreover, PPE must be put on and removed in the correct sequence to prevent contamination of skin or clothing. The importance of observing these guidelines was reinforced during the SARS (severe acute respiratory syndrome) epidemic in 2003 when some healthcare workers contracted infections, possibly from the improper use of PPE (Hon et al., 2008).

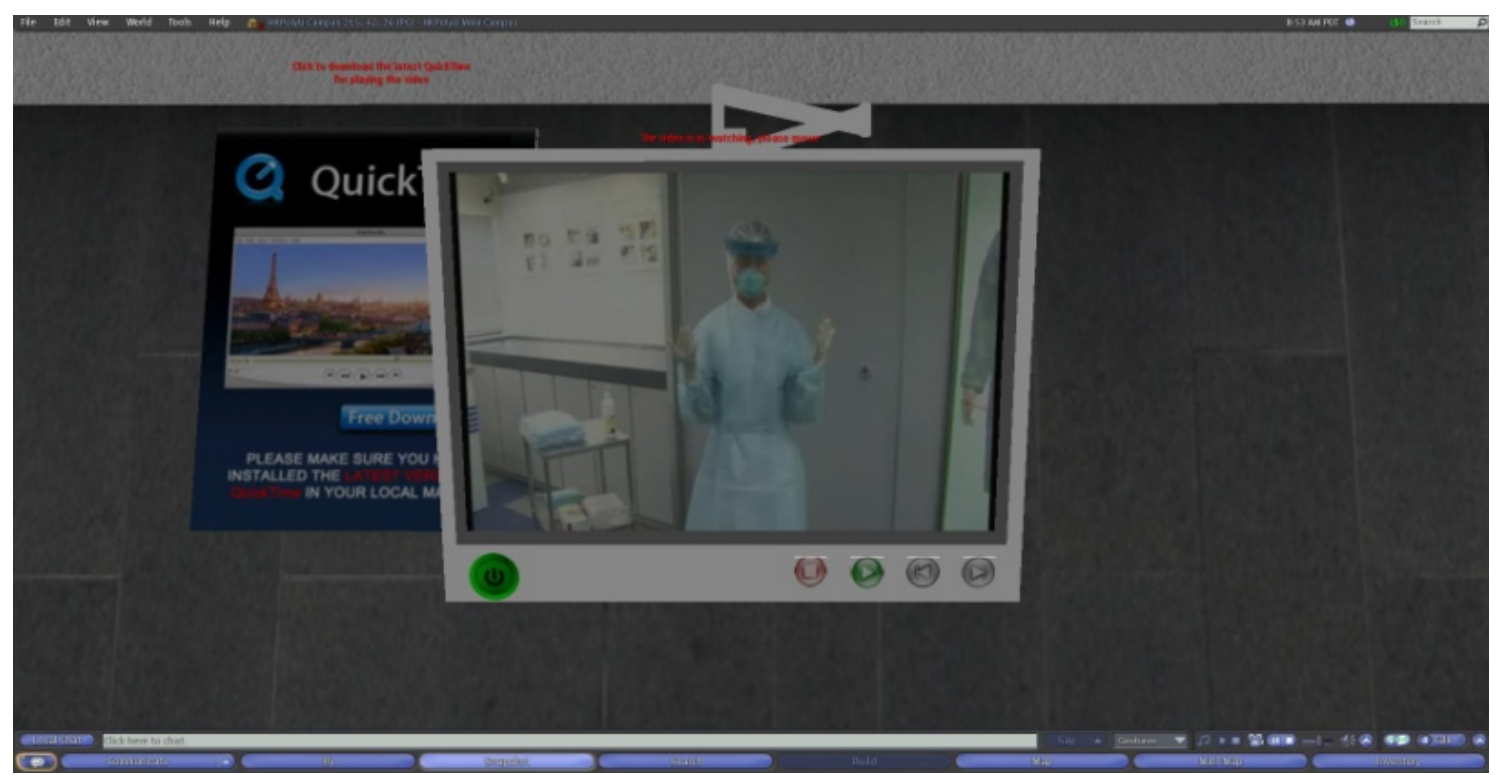

Figure 1. A video showing how to use personal protective equipment in a virtual classroom

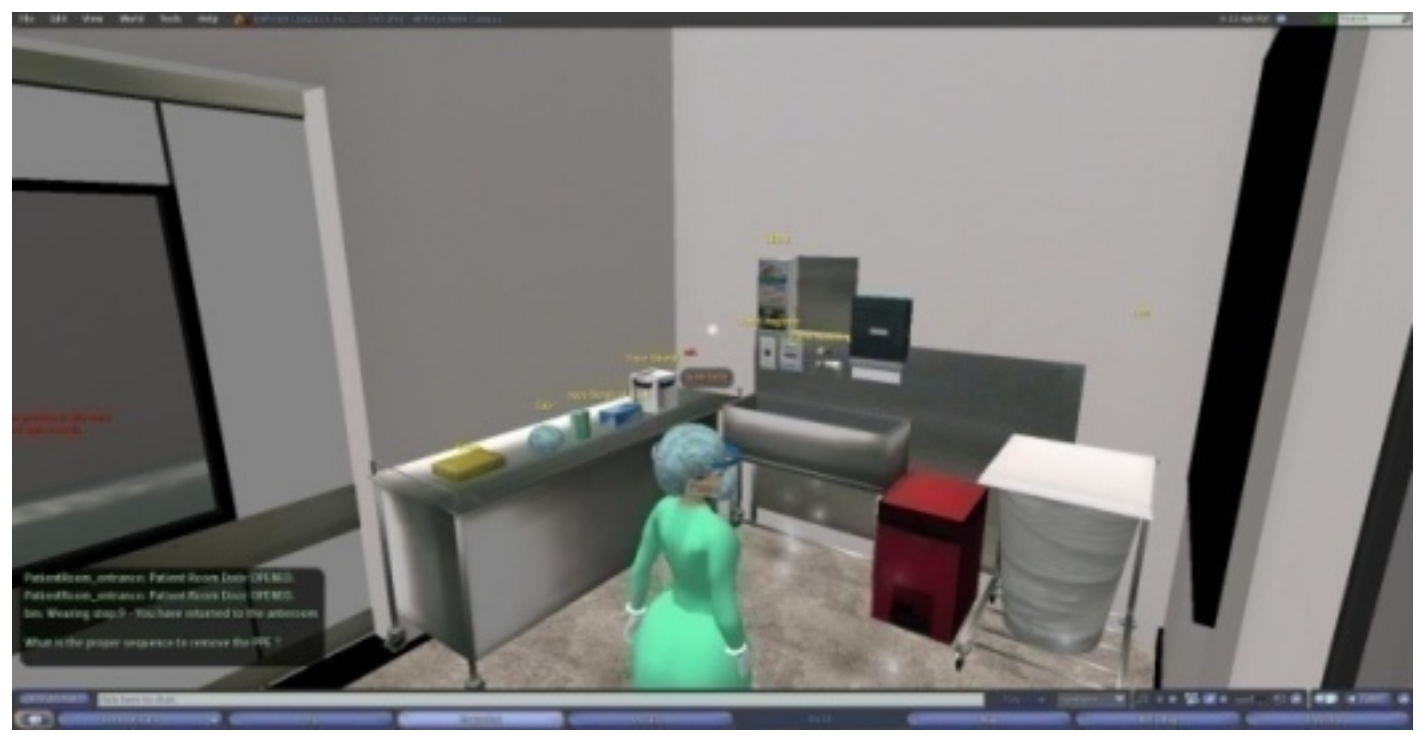

Figure 2. A virtual ward for practising the selection and use of PPE

The aim of using a 3D virtual world (PPE in SL) was to train nursing students on how to select and use PPE to protect themselves from exposure to microbiological hazards. Student avatars were required to follow proper procedures before entering the ward or any of the treatment areas. They first entered the ward through a virtual dressing room, in which they had to change into hospital uniforms and wear face masks. There were 10 single 
patient rooms in the virtual ward. Student avatars could communication with one another in the ward. However, each room only allowed one avatar to practise at a time. Inside the room, there was an anteroom for donning and removing PPE. The treatment area was designed with a patient figure lying on a bed. Before entering a treatment area, the avatar had to read the patient record and then, based on the information provided, identify the appropriate PPE to wear. The avatar had to don additional PPE items in sequence on top of the hospital uniform before being allowed to enter the treatment area. A range of PPE including gloves, gowns, face shields, caps, and masks ready to be used was arranged on a bench in the anteroom. The avatar had to click the appropriate items and add them to the inventory before wearing them. The avatar would be prompted to check if appropriate PPE had been donned before they walked to the treatment area. When leaving the treatment area, the avatar needed to remove the PPE items in sequence and then perform handwashing. Once the student had completed the entire procedure in the patient room, the student's performance was saved in a log file. The system would then automatically generate an evaluation report of his or her performance in the simulation scenario. A total of 10 different scenarios were available for nursing students to practise in the virtual ward.

\section{Research model and hypotheses}

The determinants of presence in this study are derived with reference to the conceptual model developed by Dunnington (2012), namely the nature and determinants of presence model (NDPM). Using the grounded theory approach, Dunnington examined the nature of presence in baccalaureate nursing students participating in high fidelity human patient simulation (HF-HPS). Scenario-based HF-HPS is an augmented virtuality, that is, a virtual world with added real elements on the virtuality continuum (Dunnington, 2012; Milgram \& Kishino, 1994). In her study, Dunnington observed a total of 36 simulation encounters, followed by interviews with 60 nursing students. The results suggest that the nature of presence is a dynamic state of being with a centricity between the simulation environment and the physical environment where students perceived the stimuli from one environment as salient over the other. Centricity is defined as the locus of the state of being in interaction based on the salience of perception of stimuli from the virtual environment in relation to the physical environment. Participants may centre their presence primarily inside the virtual simulation (endocentricity) or outside the virtual simulation interacting more with the physical environment (exocentricity) (Dunnington, 2014). Based on the study data, pedagogical, individual, and group factors are theorised to be determinative of the centricity of presence, which in turn is theorised to impact learning outcomes. The pedagogical factors found in her study were simulation design, stream of stimuli, and instructional process. Personality characteristics, referential experiences, preconceptions, emotional responses, and entry competencies were the individual factors. Group factors, which included group dynamics and group structure, were also found to be determinative of the sense of presence in the simulation encounters. The NDPM is particularly useful because it integrates the determinants of presence, centricity of presence, and learning outcome in one coherent framework and is rooted in empirical reality. In addition, the determinants of presence, that is, pedagogical, individual, and group factors, are theorised to be predictors of presence rather than the essence of the presence construct itself. This conceptualisation is in line with the studies of several key authors of presence (Lee, 2004; Lombard \& Ditton, 1997; Witmer, Jerome, \& Singer, 2005). The proposition of the NDPM is that understanding and managing determinants can shape changes in ways that enhance the learner's experience of presence.

Owing to the promise held by the NDPM, there is a heightened need for simplified, easily interpretable, and communicable determinants of presence. The determinants of presence in the NDPM are comprehensive, and they are in operation before and/or during the mediated learning experience. However, the relationship between the determinants of the NDPM and how they interact to affect presence were not explored in the empirical study. Similar to the NDPM, the conceptualisation of presence in this study regards self presence as the essence of the construct, and other variables such as individual or user factors as determinants of presence. The determinants are predictors of presence rather than a measure of presence itself.

Turner (2007) contended that to experience is to experience something and ordinarily these somethings are objects or events in the world. However, in presence research these are presented by way of technology. To understand an experience, it is necessary to understand both the psychological and bodily states and the objects and events which bring about the experience. Most, if not all, of these psychological and bodily factors are 
intentional, and the sense of presence is the product of an intentionality-affordance dynamic (Turner, 2007). One of the most popular research models that is related to both intention and technology is the technology acceptance model (TAM). It was proposed by Davis (1989) to explain potential users' behavioural intention to use a technology. If users lack the intention to engage in interaction with a virtual environment, it is likely that they would feel present in the immediate physical environment (exocentricity) rather than in a mediated environment (endocentricity). TAM conceptualises that an individual's intention to use a technology is determined by two beliefs: perceived usefulness and perceived ease of use. Perceived usefulness has been theorised to be influenced by various external variables, such as perceived enjoyment, cognitive style, computer self-efficacy, and subjective norm (Davis, Bagozzi, \& Warshaw, 1989; Lee, Cheung, \& Chen, 2005).

This study explored four determinants of presence: perceived usefulness (PU), which is defined as the degree to which a user views the technology as offering better value than alternative methods of carrying out the same task; perceived ease of use (PEOU), which is the extent to which a user views the usage of the technology to be relatively free of effort; computer self-efficacy (CSE), which represents a user's belief about his/her ability to perform a specific task using the technology; and subjective norm (SN), which refers to the degree to which a user perceives the demands of significant others on him/her to use the technology (Davis et al., 1989; Venkatesh \& Davis, 2000). These variables are in line with the individual (PU, PEOU, and CSE), and group (SN) determinants of presence of the NDPM. Previous research has empirically validated that PEOU, CSE, and SN have positive effects on PU (Chow et al., 2012; Kowitlawakul, 2011; Ong \& Lai, 2006; Ong, Lai, \& Wang, 2004; Venkatesh \& Davis, 2000); hence, they are theorised as determinants of PU in this study. In addition, research on computer self-efficacy suggests that low self-efficacy is associated with increased anxiety when performing a computer task (Brosnan, 1998). It is thus expected that users with low virtual world self-efficacy will be more anxious, which may directly affect their sensorial immersion and sense of presence in virtual worlds. Hence, CSE is theorised as a determinant of presence. Figure 3 provides a conceptual map of the research model examined in this study. Five research hypotheses were proposed to explore the determinants of presence:

- $\mathrm{H}_{1}$ : The subjective norm of nursing students has a positive effect on the perceived usefulness of learning selection and use of PPE in SL.

- $\quad \mathrm{H}_{2}$ : Nursing students' perception of its ease of use has a positive effect on the perceived usefulness of learning selection and use of PPE in SL.

- $\mathrm{H}_{3}$ : The computer self-efficacy of nursing students has a positive effect on the perceived usefulness of learning selection and use of PPE in SL.

- $\mathrm{H}_{4}$ : The computer self-efficacy of nursing students has a positive effect on the sense of presence in learning selection and use of PPE in SL.

- $\mathrm{H}_{5}$ : Nursing students' perception of its usefulness has a positive effect on the sense of presence in learning selection and use of PPE in SL.

\section{Instrument}

The survey instrument consisted of 14 items (Table 1) to measure five constructs of the research model. These items were adapted from previously validated instruments and modified to fit the specific technology, that is, the selection and use of PPE in SL, of the present study. SN was measured using two items adapted from Taylor and Todd (1995). PEOU was based on the instrument introduced by Venkatesh and Davis (2000) and consisted of three items. CSE contained two items adapted from Vijayasarathy (2004). The PU construct of the model was made up of four items, which were adapted from Venkatesh and Davis (2000). Finally, the self-presence construct was adapted from Jin and Park (2009) and contained three items. All the items were measured on a seven-point Likert scale ranging from 1 strongly agree to 7 strongly disagree.

\section{Sample and data collection}

Having obtained ethical approval from the institution, nursing students enrolled in undergraduate nursing programs at the School of Nursing were approached and invited to participate in hands-on workshops tailored 
to this study. Since SL was new to the students, the students were taught how to create and manipulate their own avatars and interact in the virtual world. Afterwards, they started working with their student avatars and went through the simulated patient scenarios on the selection and use of PPE. At the conclusion of the workshops, feedback was collected from students using the survey instrument. A total of 185 students joined the workshops and returned the instrument. The participants were between 20 and 24 years of age, and the majority of them were female (67.6\%). Of the participants, 22 (12\%) were first-year nursing students, 120 (65\%) were second-year nursing students, and 43 (23\%) were final-year nursing students. A total of 121 (65\%) nursing students had no previous experience with 3D games or animation.

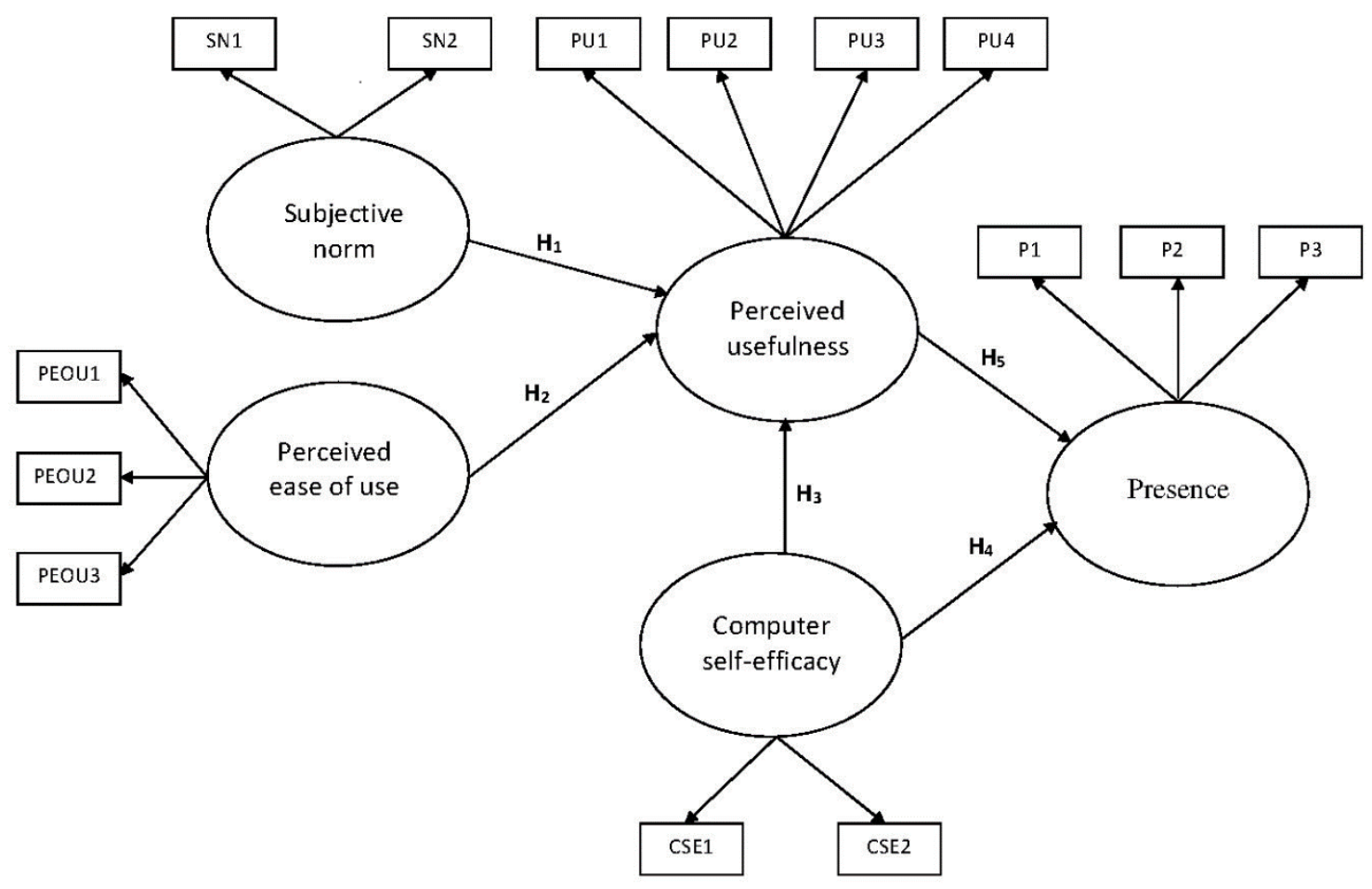

Figure 3. Research model and hypotheses

\section{Data analysis and results}

This study employed the structural equation modelling (SEM) approach to analyse the research model. SEM is a promising analytical technique for testing models because measurement errors can be controlled using a latent factor model. SEM allows for a simultaneous examination of a system of hypothesised equations involving multiple endogenous variables along with the measurement errors to provide a better understanding of the variance accounted for by the exogenous variables. It also provides multivariate goodness-of-fit indices for assessing the relationship between the variables (Kline, 2010).

The descriptive statistics of all of the variables included in the research model are shown in Table 2 . The results indicate that the students perceived that people who were important to them thought that they should use PPE in SL, that PPE in SL was easy to use and useful to them, and that the students felt confident about working with computers. However, they remained neutral regarding the sense of presence in using PPE in SL. Table 3 shows the result of a principal component analysis with a varimax rotation to identify the underlying factor structure of the observed variables. The exploratory factor analysis generated five factors with factor loadings ranging from 0.55 to 0.88 , which were well above the threshold value of 0.4 for retention (Nunnally \& Bernstein, 1994). The total variance explained by the five factors was $81.63 \%$. 
Table 1

Survey items

Subjective norm

SN1 People who influence my behaviour think that I should use PPE in Second Life.

SN2 People who are important to me think that I should use PPE in Second Life.

Perceived ease of use

PEOU1 It is easy to operate PPE in Second Life and get it to do what I want it to do.

PEOU2 I find that PPE in Second Life is very easy to use.

PEOU3 I find that the computer interface of PPE in Second Life is clear and easy to understand.

Computer self-efficacy

CSE1 I expect to become proficient in using PPE in Second Life.

CSE2 I would feel confident that I can use PPE in Second Life.

Perceived usefulness

PU1 PPE in Second Life can improve my learning efficiency.

PU2 PPE in Second Life can enhance my learning performance.

PU3 PPE in Second Life increases my learning output.

PU4 I find that PPE in Second Life is useful for my learning.

\section{Presence}

P1 While you were using PPE in Second Life, how much did you feel as if you were walking when your own avatar walked?

P2 While you were using PPE in Second Life, how much did you feel as if you were actually inside the ward when your avatar visited the ward?

P3 While you were using PPE in Second Life, how much did you feel as if you were moving when your own avatar moved?

A two-step approach of SEM analysis was undertaken to test the hypotheses in this study. The first step was to test the measurement model to ensure that the chosen indicators for a construct were reliable and valid before proceeding to the second step of testing the specified hypotheses in the structural model (Anderson \& Gerbing, 1988). The AMOS 19.0 software was used for the SEM analysis in this study.

\section{Measurement model}

The measurement model, which consists of five latent variables, namely SN, PEOU, CSE, PU, and presence, was validated by means of confirmatory factor analysis. Fit indices are used to determine the fit of a model, and combined fit indices are recommended for evaluating models (Hu \& Bentler, 1999). Fit indices are classified into three categories: absolute, parsimonious, and comparative (Brown, 2006; Hair, Black, Babin, Anderson, \& Tatham , 2006). Absolute fit indices (e.g., a normed chi-square - i.e., the ratio of $\chi^{2}$ to its degree of freedom, the $\chi^{2} / \mathrm{df}$ ratio) assess how well a model reproduces the observed data. Parsimonious indices (e.g., root mean square error of approximation, RMSEA) are similar to the absolute fit indices except that parsimonious indices also take the complexity of the model into account. Finally, the incremental fit indices (e.g., normed fit index, NFI; non-normed fit index, NNFI; and comparative fit index, CFI) measure how well a specified model fits relative to an alternative baseline model. Overall, the measurement model in this study showed a good modeldata fit: $\chi^{2} / \mathrm{df}$ ratio $=1.78$; RMSEA $=0.06$; NFI $=0.96$; NNFI $=0.97$; and CFI $=0.98$. All of the model-fit indices exceeded their respective common acceptance levels suggested by previous research (Gefen, Straub, Boudreau, 2000; Tung \& Chang, 2008; Wu, Shen, Lin, Greenes, \& Bates, 2008).

The measurement model was further assessed in terms of its reliability, convergent validity, and discriminant validity. Table 2 shows the composite reliability (CR) and Cronbach's alpha of all of the constructs in the model. 
The findings suggest good reliabilities for all the observed variables, as their coefficients exceeded the acceptable criterion of 0.60 (Bagozzi \& Yi, 1988; Fornell \& Larcker, 1981). The main difference between Cronbach's alpha and CR is that the former assumes each item to be equally weighted in the composite determination, while the latter takes into account the actual factor loadings of each individual item in the estimation. Unidimensionality and average variance extracted (AVE) were employed to evaluate convergent validity in this study (Bagozzi \& Yi, 1988). To establish unidimensionality, both the factor loading and $t$-value of items should be significant and exceed the recommended benchmarks of 0.50 and 1.96, respectively (Hair, Anderson, Tatham, \& Black, 1992). Table 2 indicates that the factor loadings of the items in the research model ranged from 0.80 to 0.97 and were significant at the level of $p<0.001$. In addition, the AVEs were all above the threshold value of 0.50 . This means that the hypothesised factors accounted for more than one half of the variances observed in their measurement items (Hair et al., 1992).

Table 2

Descriptive statistics, average variance extracted (AVE), composite reliability (CR) and factor loading of construct measurement

\begin{tabular}{|c|c|c|c|c|c|c|c|}
\hline Variables & Mean* & $\mathrm{SD}$ & $\begin{array}{c}\text { Factor } \\
\text { loading }\end{array}$ & t-value & AVE & CR & Alpha \\
\hline Subjective norm & 3.86 & 1.06 & & & 0.87 & 0.93 & 0.94 \\
\hline SN1 & 3.83 & 1.03 & 0.96 & --- & & & \\
\hline SN2 & 3.88 & 1.09 & 0.93 & 18.44 & & & \\
\hline Perceived ease of use & 3.69 & 1.25 & & & 0.83 & 0.93 & 0.96 \\
\hline PEOU1 & 3.71 & 1.32 & 0.88 & --- & & & \\
\hline PEOU2 & 3.85 & 1.29 & 0.93 & 17.39 & & & \\
\hline PEOU3 & 3.51 & 1.15 & 0.80 & 13.87 & & & \\
\hline Computer self-efficacy & 3.62 & 1.29 & & & 0.74 & 0.86 & 0.90 \\
\hline CSE1 & 3.70 & 1.29 & 0.96 & --- & & & \\
\hline CSE2 & 3.54 & 1.29 & 0.81 & 11.92 & & & \\
\hline Perceived usefulness & 3.34 & 1.28 & & & 0.71 & 0.82 & 0.88 \\
\hline PU1 & 3.32 & 1.31 & 0.92 & --- & & & \\
\hline PU2 & 3.29 & 1.28 & 0.94 & 23.81 & & & \\
\hline PU3 & 3.30 & 1.21 & 0.90 & 20.78 & & & \\
\hline PU4 & 3.43 & 1.32 & 0.92 & 21.39 & & & \\
\hline Presence & 4.07 & 1.36 & & & 0.83 & 0.91 & 0.95 \\
\hline P1 & 4.03 & 1.35 & 0.88 & --- & & & \\
\hline P2 & 4.08 & 1.35 & 0.93 & 19.72 & & & \\
\hline P3 & 4.11 & 1.38 & 0.97 & 21.79 & & & \\
\hline
\end{tabular}

Discriminant validity is assured if the square root of the AVE for a given construct is larger than the correlation between that construct and all other constructs (Fornell \& Larcker, 1981; Kerlinger \& Lee, 2000). This means that a latent variable is able to account for more variance in the observed variables associated with it than other constructs within the research model. The correlation matrix in Table 4 shows that the square roots of the AVEs are larger than the off-diagonal elements in the corresponding rows and columns, thus indicating good discriminant validity at the construct level in this study. 
Table 3

Results of exploratory factor analysis

\begin{tabular}{lrrrrr}
\hline & Factor & & & & \\
\cline { 2 - 6 } & 1 & 2 & 3 & 4 & 5 \\
\hline Subjective norm & & & & & \\
SN1 & 0.36 & .027 & 0.16 & 0.11 & $\mathbf{0 . 8 3}$ \\
SN2 & 0.36 & 0.24 & 0.14 & 0.14 & $\mathbf{0 . 8 3}$ \\
Perceived ease of use & & & & & \\
$\quad$ PEOU1 & 0.54 & $\mathbf{0 . 5 5}$ & 0.30 & 0.03 & 0.09 \\
PEOU2 & 0.56 & $\mathbf{0 . 5 9}$ & 0.24 & 0.03 & 0.08 \\
PEOU3 & 0.54 & $\mathbf{0 . 6 3}$ & 0.14 & 0.01 & 0.06 \\
Computer self-efficacy & & & & & \\
CSE1 & 0.33 & 0.16 & 0.32 & $\mathbf{0 . 7 9}$ & 0.10 \\
CSE2 & 0.10 & 0.27 & 0.35 & $\mathbf{0 . 7 4}$ & 0.09 \\
Perceived usefulness & & & & & \\
PU1 & $\mathbf{0 . 7 8}$ & 0.24 & 0.27 & 0.14 & 0.25 \\
PU2 & $\mathbf{0 . 7 5}$ & 0.27 & 0.29 & 0.08 & 0.32 \\
PU3 & $\mathbf{0 . 7 2}$ & 0.32 & 0.29 & 0.04 & 0.25 \\
PU4 & $\mathbf{0 . 7 9}$ & 0.29 & 0.26 & 0.13 & 0.21 \\
Presence & & & & & \\
P1 & 0.34 & 0.14 & $\mathbf{0 . 8 2}$ & 0.21 & 0.15 \\
P2 & 0.25 & 0.15 & $\mathbf{0 . 8 8}$ & 0.21 & 0.10 \\
P3 & 0.29 & 0.14 & $\mathbf{0 . 8 5}$ & 0.28 & 0.11 \\
\hline
\end{tabular}

Corresponding factor loadings of the items are in italic type.

Table 4

Square root of average variance extracted (AVE) and correlations of all constructs

\begin{tabular}{llrrrrr}
\hline & & 1 & 2 & 3 & 4 & 5 \\
\hline 1 & Subjective norm & $\mathbf{0 . 9 3}$ & & & & \\
2 & Perceived ease of use & 0.59 & $\mathbf{0 . 8 6}$ & & & \\
3 & Computer self-efficacy & 0.45 & 0.48 & $\mathbf{0 . 8 4}$ & & \\
4 & Perceived usefulness & 0.67 & 0.73 & 0.56 & $\mathbf{0 . 9 1}$ & \\
5 & Presence & 0.44 & 0.51 & 0.65 & 0.61 & $\mathbf{0 . 9 1}$ \\
\hline
\end{tabular}

Square roots of AVEs are shown as diagonal elements in bold type. The diagonal elements were greater than the corresponding off-diagonal elements in the same row and column, indicating the discriminant validity.

\section{Structural model}

In this study, the structural model consisted of three exogenous variables (i.e., SN, PEOU, and CSE) and two endogenous variables (i.e., PU and presence). Similar to the measurement model, a test of the structural model by comparing the combined fit indices with their corresponding recommended values revealed a good model fit $\left(\chi^{2} / \mathrm{df}\right.$ ratio $\left.=1.74 ; \mathrm{RMSEA}=0.06 ; \mathrm{NFI}=0.96 ; \mathrm{NNFI}=0.97 ; \& \mathrm{CFI}=0.98\right)$.

Figure 4 shows the properties of the causal paths, including standardised path coefficients, $p$-values, and variance explained, for each equation in the hypothesised model. All of the hypotheses were supported since their respective path coefficients were significant at the level of $p<0.001$. SN ( $\beta=0.32, p<0.001)$, PEOU ( $\beta$ $=0.44, p<0.001)$, CSE $(\beta=0.21, p<0.001)$ had a significant positive effect on PU - together they accounted for $65 \%$ of the variance in PU. Both PU $(\beta=0.36, p<0.001)$ and CSE $(\beta=0.45, p<0.001)$ had a significant 
positive effect on presence. The research model explained 52\% of the variance in the students' experience of presence in using PPE in SL.

Table 5 presents the direct, indirect, and total effects of SN, PEOU, CSE, and PU on presence. Among all of the predictor variables, CSE exhibited the strongest total effect on presence (0.53), followed by PU (0.36). It is worth noting that PEOU exhibited a strong direct effect on PU (0.44), followed by subjective norm $(0.32)$ and CSE (0.21).

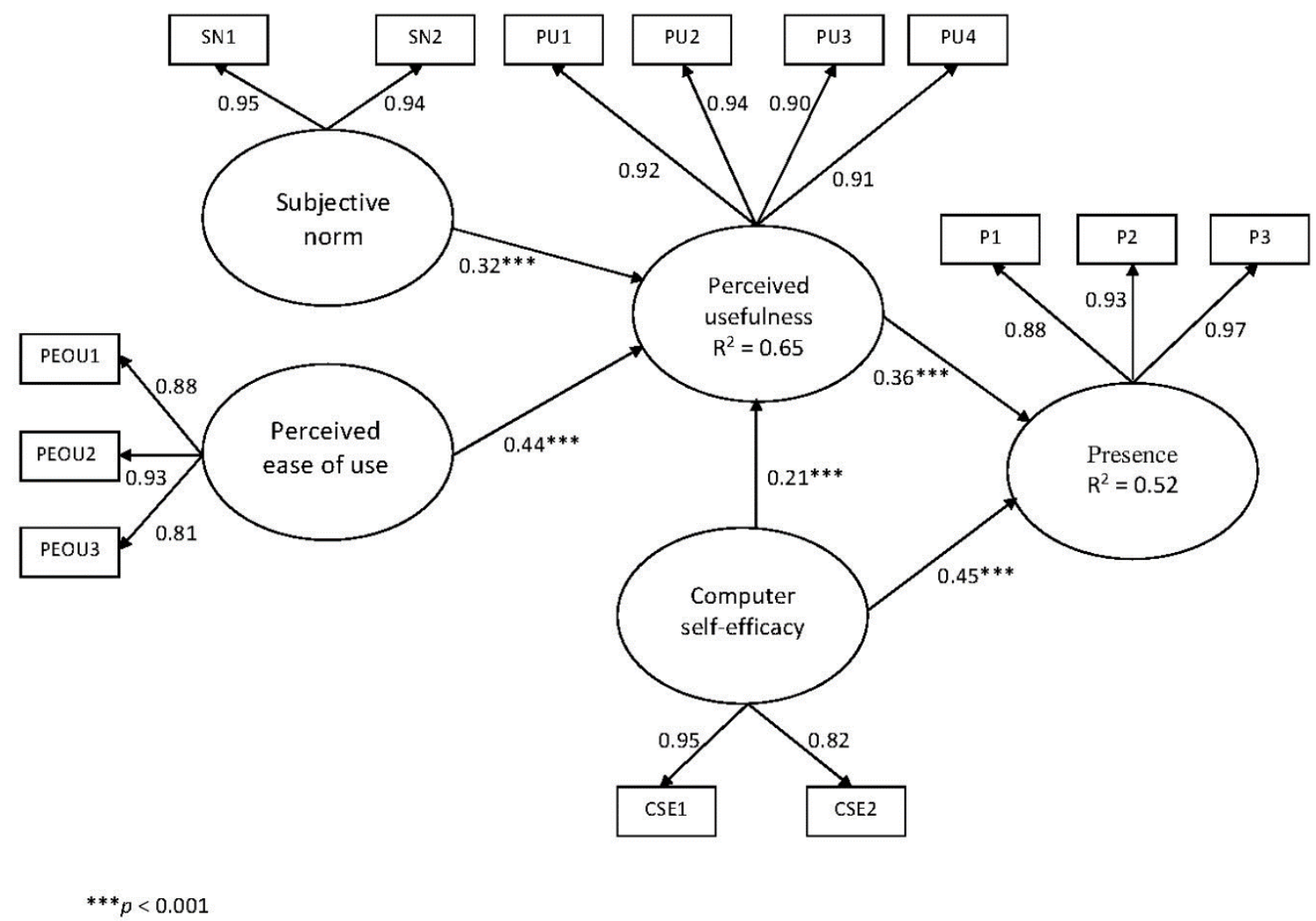

Figure 4. Results of SEM testing

Table 5

The direct, indirect, and total effects of variables on presence

\begin{tabular}{|c|c|c|c|c|c|c|}
\hline & \multicolumn{2}{|c|}{ Direct effect } & \multicolumn{2}{|c|}{ Indirect effect } & \multicolumn{2}{|c|}{ Total effect } \\
\hline & PU & $\mathrm{P}$ & $\mathrm{PU}$ & $\mathrm{P}$ & PU & $\mathrm{P}$ \\
\hline SN & 0.32 & & & 0.12 & 0.32 & 0.12 \\
\hline PEOU & 0.44 & & & 0.16 & 0.44 & 0.16 \\
\hline CSE & 0.21 & 0.45 & & 0.08 & 0.21 & 0.53 \\
\hline PU & & 0.36 & & & & 0.36 \\
\hline
\end{tabular}

SN, subjective norm; PEOU, perceived ease of use; CSE, computer self-efficacy; PU, perceived usefulness; $\mathrm{P}$, presence. 


\section{Discussion}

This study explored the effects of SN, PEOU, CSE, and PU on the students' experience of presence in a 3D virtual learning environment. Using structural equation modeling techniques, the research model (Figure 3) was tested, and the findings indicated a good fit with the data. The measurement model was confirmed with adequate reliability, convergent validity, and discriminant validity. In addition, all path coefficients in the structural model were significant at the $p<0.001$ level. The effect of CSE on presence was greater than that of PU. Perceived usefulness was in turn influenced by SN, PEOU, and CSE - together they explained 65 percent of the variance in PU. All hypotheses in the research model were supported, and the results of this study show that SN, PEOU, CSE, and PUaccount for 52\% of the total variance of the sense of presence in the 3D virtual world.

The results of this study reveal that CSE has the strongest direct influence on the level of presence experienced by the students. Students with higher CSE are likely to experience a higher level of presence in the virtual world. This suggests that students' confidence in using technology and their feeling of control play a significant role in the experience of presence. Cognitive load theory, which was developed by Sweller (1988), provides a possible explanation for this. SL is a different type of technology and new to the students. It requires an extraneous cognitive load to use the avatar to navigate and interact in the 3D virtual environment. Students who were less confident in their computer skills might have found the PPE in SL so complex that they did not have sufficient cognitive resources left to immerse themselves and engage in the activities that were provided. If the students' sense of presence is to be enhanced, their computer skills need to be further developed and the technical challenges must be dealt with. Hence, students to whom the use of the virtual world is a new experience should be provided with longer introductory sessions before being allowed to attempt the simulation. Both on- and off-line support services must also be enhanced to boost the self-confidence of students in using the system.

PU was found to be a significant antecedent to the sense of presence. This implies that if students find PPE in SL to be useful, their experience of presence would be enhanced. The use of PPE is an essential measure to protect healthcare workers from contracting infectious diseases in clinical settings. However, nursing students are particularly at risk because of inexperience and lack of knowledge in using PPE. In this study, students in general found PPE in SL to be useful, even though their use of the system was voluntary. The virtual classroom and, in particular, the simulation case scenarios in the virtual hospital wards allow students to learn and practice the selection and use of PPE without the risk of exposure to infections. The findings of this study suggest that to enhance the experience of presence, emphasis should be placed on increasing the PU of virtual worlds, which is possible through improving SN, PEOU, and CSE of students. Presence is a matter of focus that occurs when we direct our attention towards something. Experiencing presence in a virtual world requires us to focus on one meaningful coherent set of stimuli to the exclusion of unrelated stimuli in the physical environment. If we find a virtual world to be useful and feel confident in using it, we will be more willing to suspend our disbelief and allocate attentional resources to the mediated environment.

The results of this study corroborate those of Chow et al. (2012), Ong and Lai (2006), Ong, Lai, and Wang (2004), Kowitlawakul (2011), and Venkatesh and Davis (2000) in that SN, PEOU, and CSE were found to have direct positive effects on PU. As depicted in Figure 4, PEOU was found to be the strongest predictor of PU, followed by SN, and then CSE. This means that the students' perception of how easy it is to use the system plays a very important role in enhancing the experience of presence through PU. This suggests that when introducing the PPE in SL, strategies should be devised to ensure that the system is easy and relatively effortless to use. These include designing user-friendly interfaces, hiding the complexity and details of the hardware and software involved, and simplifying the students' access to the virtual wards and performance of tasks through changes in the design of the virtual environment. The usefulness of the system hinges on the way in which the system is designed and the extent to which students perceive the system easy to use. If students are struggling, they may actually believe that the system is too difficult to use and that the benefits of using PPE in SL are outweighed by the effort required to use it. 
SN was found to have a direct effect on PU, which in turn impacts on the experience of presence in the PPE in SL. In this study, it is possible that at the undergraduate level, the nursing students observed and treated significant others as models of technology use. These included fellow nursing students, staff nurses, and nurse educators. The nursing students in this study might have considered technology use to be important from their experiences during nursing training. Currently, information and communication technology is widely used to support nursing education, clinical practices, administration, and research. For example, nursing students have to use Blackboard for managing their learning activities and Electronic Health Records for documenting the clinical conditions of their patients. Hence, nurse educators can promote the use of the PPE in SL by making explicit the advantages of independent learning in selecting and using PPE in the 3D virtual environment. Besides PEOU and SN, CSE also has a significant impact on PU. When nursing students believe themselves to be capable of using the PPE in SL and at the same time perceive that the system is easy to use and that people who are significant to themselves expect them to use it, they are likely to form a strong belief that the system is useful.

Presence depends on how well we are connected to or involved in a virtual world. This study contributes to the existing research on presence by providing added insights into the predictive ability of the four user characteristics, namely CSE, PU, PEOU, and SN, to influence the sense of presence in virtual worlds. Despite the application of rigorous procedures, the findings of this study should be considered in light of its limitations. First, this is the first test of the research model, which should be subjected to further testing with different participants and contexts. Second, this study was conducted in a single university, thus limiting the generalisability of the findings to other students. Third, participation in this study was voluntary, and students who were interested in virtual worlds were more likely to respond. As such, the data might have been affected by the self-selection biases of the participants. In addition, this study used a cross-sectional design. Given that perceptions are shaped by one's experiences and change over time, a longitudinal study with measures at different times will be helpful to enhance our prediction and understanding of the causality and interrelationships between the variables over time. The research model in this study is parsimonious and provides a starting point for model extension. Further studies may explore if other constructs such as hedonic consumption (Holsapple \& Wu, 2007) can further enhance the model.

\section{Conclusions}

Virtual worlds such as SL provide unique opportunities to simulate real-life scenarios and are showing potential as an effective platform for healthcare education (Chow et al., 2012; Skiba, 2009). As learning using this innovative technology continues to grow each year, the construct of presence will become even more important. This study makes a significant contribution by developing and validating an initial theoretical model of the determinants of presence in a 3D virtual world. SEM was employed as the main technique for data analysis to ensure methodological rigor. The theoretical constructs and their relationships in the model have been identified and systematically and empirically tested. The findings from this study suggest how the sense of presence occurred in the virtual environment. This model is intended to guide future efforts to develop virtual worlds. However, it should be noted that direct experimental tests of the implications need to be conducted.

\section{References}

Alsina-Jurnet, I., \& Gutierrez-Maldonado, J. (2010). Influence of personality and individual abilities on the sense of presence experienced in anxiety triggering virtual environments. International Journal of HumanComputer Studies, 68(10), 788-801. doi:10.1016/j.ijhcs.2010.07.001

Anderson, J. C., \& Gerbing, D. W. (1988). Structural equation modeling in practice: A review and recommended two-step approach. Psychological Bulletin, 103(3), 411-423. doi:10.1037/00332909.103.3.411

Bagozzi, R. P., \& Yi, Y. (1988). On the evaluation of structural equation models. Journal of the Academy of Marketing Science, 16(1), 74-94. doi:10.1007/BF02723327

Bell, M. (2008). Toward a definition of "virtual worlds". Journal of Virtual Worlds Research, 1(1). Retrieved from https://journals.tdl.org/jvwr/index.php/jvwr/article/viewFile/283/237 
Blascovich, J., \& Bailenson, J. (2011). Infinite reality: Avatars, eternal life, new worlds, and the dawn of the virtual revolution [Kindle DX version]. New York, NY: HarperCollins e-books.

Brosnan, M. J. (1998). The impact of computer anxiety and self-efficacy upon performance. Journal of Computer Assisted Learning, 14(3), 223-234. doi:10.1046/j.1365-2729.1998.143059.x

Brown, T. (2006). Confirmatory factor analysis for applied research. New York, NY: Guildford Press.

Bulu, S. T. (2012). Place presence, social presence, co-presence, and satisfaction in virtual worlds. Computers \& Education, 58(1), 154-161. doi:10.1016/j.compedu.2011.08.024

Casanova, L., Alfano-Sobssey, E., Rutala, W. A., Weber, D., \& Sobsey, M. (2008). Virus transfer from personal protective equipment to healthcare employees' skin and clothing. Emerging Infectious Diseases, 14(8), 1291-1293. doi:10.3201/eid1408.080085

Chow, M., Herold, D. K., Choo, T. M., \& Chan, K. (2012). Extending the technology acceptance model to explore the intention to use Second Life for enhancing healthcare education. Computers \& Education, 59(4), 1136-1144. doi:10.1016/j.compedu.2012.05.011

Dalgarno, B., Lee, M. J. W., Carlson, L., Gregory, S., \& Tynan, B. (2011). An Australian and New Zealand scoping study on the use of 3D immersive virtual worlds in higher education. Australasian Journal of Educational Technology, 27(1), 1-15. Retrieved from http://ajet.org.au/index.php/AJET/article/view/978/251

Davis, F. D. (1989). Perceived usefulness, perceived ease of use, and user acceptance of information technology. MIS Quarterly, 13(3), 318-340. doi:10.2307/249008

Davis, F. D., Bagozzi, R. P., \& Warshaw, P. R. (1989). User acceptance of computer technology: A comparison of two theoretical models. Management Science, 35(8), 982-1003. doi:10.1287/mnsc.35.8.982

deByl, P. (2009). Hybrid 2D/3D development of interactive simulations. In D. Gibson \& Y. Baek (Eds.), digital simulations for improving education: Learning through artificial teaching environments (pp. 171187). Hershey, PA: Information Science Reference. doi:10.4018/978-1-60566-322-7.ch009

Department of Health. (2001). Standard principles for preventing hospital-acquired infections. Journal of Hospital Infection, 47(supplement), S21-S37. doi:10.1053/jhin.2000.0889

Dickey, M. D. (2003). Teaching in 3D: Pedagogical affordances and constraints of 3D virtual worlds for synchronous distance learning. Distance Education, 24(1), 105-121. doi:10.1080/01587910303047

Dunnington, R. M. (2012). The nature and determinants of presence among nursing students participating in high fidelity human patient simulation (Doctoral dissertation). Retrieved from https://etd.ohiolink.edu/

Dunnington, R. M. (2014). Presence with scenario-based high fidelity human patient simulation. Nursing Science Quarterly, 27(2), 157-164. doi:10.1177/0894318414522655

Fornell, C., \& Larcker, D. F. (1981). Evaluating structural equation models with unobservable variables and measurement errors. Journal of Marketing Research, 18(1), 39-50. doi:10.2307/3151312

Galloso, I., Feijoo, C., \& Santamaria, A. (2015). Novel approaches to immersive media: From enlarged fieldof-view to multi-sensorial experiences. In A. Kondoz \& T. Dagiuklas (Eds.), Novel 3D media technologies (pp. 9-24). New York, NY: Springer. doi:10.1007/978-1-4939-2026-6_2

Gefen, D., Straub, D. W., \& Boudreau, M. C. (2000). Structural equation modeling and regression: guidelines for research practice. Communications of the Association for Information Systems, 4(7), 1-9. Retrieved from http://aisel.aisnet.org/cais/vol4/iss1/7

Girvan, C., \& Savage, T. (2010). Identifying an appropriate pedagogy for virtual worlds: A communal constructivism case study. Computers \& Education, 55(1), 342-349. doi:10.1016/j.compedu.2010.01.020

Hair, J. F., Black, W. C., Babin, B. J., Anderson, R. E., \& Tatham, R. L. (2006). Multivariate data analysis (6th ed.). Upper Saddle River, NJ: Prentice-Hall.

Hair, J. T., Anderson, R. E., Tatham, R. L., \& Black, W. C. (1992). Multivariate data analysis with readings. New York, NY: Macmillan.

Holsapple, C. W., \& Wu, J. (2007). User acceptance of virtual worlds: The Hedonic Framework. Database for Advances in IS, 38(4), 86-89. doi:10.1145/1314234.1314250

Hon, C. H., Gamage, B., Bryce, E. A., LoChang, J., Yassi, A., Maultsaid, D., \& Yu, S. (2008). Personal protective equipment in health care: Can online infection control courses transfer knowledge and improve proper selection and use? American Journal of Infection Control, 36(10), e33-e37. doi:10.1016/j.ajic.2008.07.007 
Hu, L. T., \& Bentler, P. M. (1999). Cutoff criteria for fit indexes in covariance structure analysis: Conventional criteria versus new alternatives. Structural Equation Modeling, 6(1), 1-55. doi:10.1080/10705519909540118

Ijsselsteijn, W., deRidder, H., Freeman, J., \& Avons, S. E. (2000). Presence: Concept, determinants and measurement. In B. E. Rogowitz \& T. N. Pappas (Eds.), Proceedings of the SPIE Conference, Human Vision and Electronic Imaging V (Vol. 3959, pp. 520-529). Bellingham, WA: SPIE. doi:10.1117/12.387188

Ijsselsteijn, W., \& Riva, G. (2003). Being there: The experience of presence in mediated environments. In G. Riva, F. Davide, \& W. A. Ijsselsteijn (Eds.), Being there: Concepts, effects and measurements of user presence in synthetic environments (pp. 4-16). Amsterdam: IOS Press.

Jennings, N., \& Collins, C. (2007). Virtual or virtually U: Educational institutions in Second Life. International Journal of Human and Social Sciences, 2(3), 180-186. Retrieved from http://citeseerx.ist.psu.edu/viewdoc/download?doi=10.1.1.180.3529\&rep=rep1\&type=pdf

Jin, S. A., \& Park, N. (2009). Parasocial interaction with my avatar: Effects of interdependent self-construal and the mediating role of self-presence in an avatar-based console game, Wii. CyberPsychology \& Behavior, 12(6), 723-727. doi:10.1089/cpb.2008.0289

Johnson, C. M., Vorderstrasse, A., \& Shaw, R. (2009). Virtual worlds in health care higher education. Journal of Virtual World Research, 2(2), 1-12. doi:10.4101/jvwr.v2i2.699

Kamel Boulos, M. N., Ramloll, R., Jones, R., \& Toth-Cohen, S. (2008). Web 3D for public, environmental and occupational health: Early examples from Second Life. International Journal of Environmental Research and Public Health, 5(4), 290-317. doi:10.3390/ijerph5040290

Kerlinger, F. N., \& Lee, H. B. (2000). Foundations of behavioral research. Fort Worth, TX: Harcourt College Publisher.

Kowitlawakul, Y. (2011). Technology acceptance model: Predicting nurses' intention to use telemedicine technology (eICU ${ }^{\circledR}$ ). Computers, Informatic, Nursing, 29(7), 411-418. doi:10.1097/NCN.0b013e3181f9dd4a

Kline, R. B. (2010). Principles and practice of structural equation modeling. New York, NY: Guilford Press.

Lackner, J. R., \& DiZio, P. (1998). Spatial orientation as a component of presence: Insight gained from nonterrestrial environments. Presence: Teleoperators and Virtual Environments, 7(2), 108-115. doi:10.1162/105474698565613

Lee, K. M. (2004). Presence, explicated. Communication Theory, 14(1), 27-50. doi:10.1111/j.14682885.2004.tb00302.x

Lee, M. K. O., Cheung, C. M. K., \& Chen, Z. (2005). Acceptance of internet-based learning medium: the role of extrinsic and intrinsic motivation. Information \& Management, 42(8), 1095-1104. doi:10.1016/j.im.2003.10.007

Lombard, M., \& Ditton, T. (1997). At the heart of it all: The concept of presence. Journal of ComputerMediated Communication, 3(2). doi:10.1111/j.1083-6101.1997.tb00072.x

Mikropoulos, T. A. (2006). Presence: A unique characteristic in educational virtual environments. Virtual Reality, 10(3-4), 197-206. doi:10.1007/s10055-006-0039-1

Mikropoulos, T. A., \& Natsis, A. (2011). Educational virtual environments: A ten-year review of empirical research (1999-2009). Computers \& Education, 56(3), 769-780. doi:10.1016/j.compedu.2010.10.020

Milgram, P., \& Kishino, F. (1994). A taxonomy of mixed reality visual displays. IEICE: Transactions on Information Systems, 77(12), 1321-1329. Retrieved from http://cs.gmu.edu/ zduric/cs499/Readings/r76JBo-Milgram_IEICE_1994.pdf

Minsky, M. (1980, June). Telepresence. Omni, 45-51.Retrieved from https://web.media.mit.edu/ minsky/papers/Telepresence.html

Nunnally, J. C., \& Bernstein, I. (1994). Psychometric theory. New York, NY: McGraw-Hill.

Ong, C. S., \& Lai, J. Y. (2006). Gender differences in perceptions and relationships among dominants of elearning acceptance. Computers in Human Behaviors, 22(5), 816-829. doi:10.1016/j.chb.2004.03.006

Ong, C., Lai, J., \& Wang, Y. (2004). Factors affecting engineers' acceptance of asynchronous e-learning systems in high-tech companies. Information \& Management, 41(6), 795-804. doi:10.1016/j.im.2003.08.012

Pitt, M. A., Myung, I. J., \& Zhang, S. (2002). Toward a method of selecting among computational models of cognition. Psychological Review, 109(3), 472-491. doi:10.1037/0033-295X.109.3.472 
Ribbens, W., \& Vanden Abeele, M. (2008). Socio-demographic and psychological user determinants in a gaming experience. In A. Spagnolli \& L. Gamberini (Eds.), Presence 2008: Proceedings of the 11th International Workshop on Presence (pp. 163-173). International Society for Presence Research. Retrieved from http://astro.temple.edu/ lombard/ISPR/Proceedings/2008/Ribbens.pdf

Romano, D. M., \& Brna, P. (2001). Presence and reflection in training: Support for learning to improve quality decision-making skills under time limitations. CyberPsychology \& Behavior, 4(2), 265-277. doi:10.1089/109493101300117947

Schmidt, B., \& Stewart, S. (2010). Implementing the virtual world of Second Life into community nursing theory and clinical courses. Nurse Educator, 35(2), 74-78. doi:10.1097/NNE.0b013e3181ced999

Schuemie, M. J., Van Der Straaten, P., Krijin, M., \& Van Der Mast, C. A. P. G. (2001). Research on presence in virtual reality: a survey. Cyberpsychology \& Behaviour, 4(2), 183-210. doi:10.1089/109493101300117884

Selverian, M. M, \& Hwang, H. S. (2003). In search of presence: A systematic evaluation of evolving VLEs. Presence: Teleoperators and Virtual Environments, 12(5), 512-522. doi:10.1162/105474603322761306

Siegel, J. D., Rhinehart, E., Jackson, M., Chiarello, L., \& the Healthcare Infection Control Practices Advisory Committee. (2007). Guideline for isolation precautions: Preventing transmission of infectious agents in healthcare settings. Retrieved from http://www.cdc.gov/hicpac/pdf/isolation/Isolation2007.pdf

Skiba, D. J. (2009). Nursing education 2.0: A second look at Second Life. Nursing Education Perspectives, 30(2), 129-131. doi:10.1043/1536-5026-030.002.0129

Slater, M. (2003). A note on presence terminology. Retrieved from http://s3.amazonaws.com/publicationslist.org/data/melslater/ref201/a\%20note\%20on\%20presence\%20terminology.pdf

Slater, M., \& Wilbur, S. (1997). A framework for immersive virtual environments (FIVE): Speculations on the role of presence in virtual environments. Presence, 6(6), 603-616. Retrieved from http://citeseerx.ist.psu.edu/viewdoc/download?doi=10.1.1.472.622\&rep=rep1\&type=pdf

Sweller, J. (1988). Cognitive load during problem solving: Effects on learning. Cognitive Science, 12(2), 257285. doi:10.1207/s15516709cog1202_4

Tamborini, R., \& Skalski, P. (2006). The role of presence in the experience of electronic games. In P. Vorderer \& B. Jennings (Eds.), Playing video games: Motives, responses, and consequences (pp. 225240). Mahwah, NJ: Erlbaum.

Taylor, S., \& Todd, P. A. (1995). Understanding information technology usage: A test of competing models. Information System Research, 6(2), 144-176. doi:10.1287/isre.6.2.144

Tung, F. C., \& Chang, S. C. (2008). Nursing students’ behavioral intention to use online courses: A questionnaire survey. International Journal of Nursing Studies, 45(9), 1299-1309. doi:10.1016/j.ijnurstu.2007.09.011

Turner, P. (2007). The intentional basis of presence. In L. Moreno (Ed.), Proceedings of PRESENCE 2007: The 10th Annual Workshop on Presence (pp. 127-133). International Society for Presence Research. Retrieved from http://astro.temple.edu/ lombard/ISPR/Proceedings/2007/Turner.pdf

Venkatesh, V., \& Davis, F. D. (2000). A theoretical extension of the technology acceptance model: Four longitudinal field studies. Management Science, 46(2), 186-204. doi:10.1287/mnsc.46.2.186.11926

Vijayasarathy, L. R. (2004). Predicting consumer intentions to use on-line shopping: The case for an augmented technology acceptance model. Information \& Management, 41(6), 747-762. doi:10.1016/j.im.2003.08.011

Weibel, D., Wissmath, B., \& Stricker, D. (2011). The influence of neuroticism on spatial presence and enjoyment in films. Personality and Individual Differences, 51(7), 866-869. doi:10.1016/j.paid.2011.07.011

Welch, D. J. (2008). Virtual worlds: Moving beyond today. EDUCAUSE Review, 43(5), 12-13. Retrieved from https://net.educause.edu/ir/library/pdf/ERM0858.pdf

Winn, W. (1993). A conceptual basis for educational applications of virtual reality. Washington, DC: Human Interface Technology Laboratory. Retrieved from http://www.hitl.washington.edu/research/education/winn/winn-paper.html

Witmer, B. G., Jerome, C. J., \& Singer, M. J. (2005). The factor structure of the presence questionnaire. Presence: Teleoperators and Virtual Environments, 14(3), 298-312. doi:10.1162/105474605323384654 
Wu, J. H., Shen, W. S., Lin, L. M., Greenes, R. A., \& Bates, D. W. (2008). Testing the technology acceptance model for evaluating healthcare professionals' intention to use an adverse event reporting system. International Journal for Quality in Health Care, 20(2), 123-129. doi:10.1093/intqhc/mzm074

Corresponding author: Meyrick Chow, meyrickchow@twc.edu.hk

Australasian Journal of Educational Technology (C) 2016.

Please cite as: Chow, M. (2016). Determinants of presence in 3D virtual worlds: A structural equation modelling analysis. Australasian Journal of Educational Technology, 32(1), 1-18. 